\section{Autotransfusão imediata em cão - relato de caso}

\author{
[Instant autotransfusion in dog - case report]
}

\author{
M.B. Stocco ${ }^{1}$, D.A. Sônego ${ }^{1}$, N.P. Sousa ${ }^{2}$, L.G. Gomes ${ }^{1}$, \\ S. Monzem ${ }^{1}$, A.C. Martini ${ }^{1}$, V.R.F. Sousa ${ }^{3}$, R.L. Souza ${ }^{3}$ \\ ${ }^{1}$ Aluno de pós-graduação - Universidade Federal de Mato Grosso - Cuiabá, MT \\ ${ }^{2}$ Aluno de graduação - Universidade Federal de Mato Grosso - Cuiabá, MT \\ ${ }^{3}$ Universidade Federal de Mato Grosso - Cuiabá, MT
}

S. Monzem

https://orcid.org/0000-0002-8600-4875 A.C. Martini

https://orcid.org/0000-0002-2553-3685 V.R.F. Sousa

https://orcid.org/0000-0001-6209-4514 R.L. Souza

https://orcid.org/0000-0002-8079-7181

\title{
RESUMO
}

As hemorragias podem levar à morte, em caso de não interrupção e recuperação da volemia. A substituição do sangue pode ser realizada por transfusão homóloga ou autóloga. Na transfusão homóloga, o sangue é obtido de um doador, na autotransfusão ou transfusão autóloga, o sangue é coletado do próprio paciente. Objetivou-se relatar a autotransfusão em um cão como um método simples, rápido e barato, e discutir esse procedimento como uma alternativa emergencial para clínicas e hospitais que não possuem bancos de sangue ou animais doadores. Foi atendido um cão Rottweiler fêmea, 42,8kg, 10 anos de idade, com queixa principal de tumor mamário e para realização de castração. Após o procedimento, a paciente apresentou hemoperitônio, sendo encaminhada para novo procedimento cirúrgico, durante o qual se observou grande quantidade de sangue livre na cavidade abdominal. Foi realizada a autotransfusão de emergência, pela técnica das duas seringas, devido à indi1111sponibilidade de sangue ou animal doador no momento do procedimento cirúrgico emergencial, demonstrando ser uma opção eficiente, econômica, de fácil acesso e segura, por ser uma transfusão normotérmica, apresentar diminuição do risco de sobrecarga circulatória e por ser o sangue compatível, devendo ser mais explorada, pois existem poucos dados descritos na literatura.

Palavras-chave: hemoperitônio, transfusão autóloga, hemorragia

\begin{abstract}
Hemorrhages can lead to death in case of non-interruption and recovery of blood volume. Blood replacement may be performed by homologous or autologous transfusion. In homologous transfusion, blood is obtained from a donor, in autotransfusion or autologous transfusion, blood is collected from the patient himself. We aimed to report autotransfusion in a dog as a simple, quick, and inexpensive method, and discuss it as an emergency alternative for clinics and hospitals that do not have blood banks or animal donors. A female Rottweiler dog, $42.8 \mathrm{~kg}, 10$ years of age, was treated with a primary complaint of mammary tumor and for castration. After the procedure the patient presented hemoperitoneum, being referred to a new surgical procedure, where a large amount of free blood was observed in the abdominal cavity. The emergency autotransfusion was performed by the two syringes technique, due to the unavailability of blood or donor animal at the time of the emergency surgical procedure, proving to be an efficient, economical, easily accessible and safe option because it is a normothermic transfusion, risk of circulatory overload and blood is compatible. It should be more exploited because there are few data described in the literature.
\end{abstract}

Keywords: hemoperitoneum, autologous transfusion, hemorrhage

Recebido em 18 de dezembro de 2017

Aceito em 12 de março de 2019

E-mail: matiasnave@hotmail.com 


\section{INTRODUÇÃO}

As hemorragias podem levar a óbito, caso não ocorra a interrupção e a recuperação da volemia adequada (Robinson et al., 2016). Para tanto, a reposição sanguínea pode ser realizada com a transfusão homóloga ou autóloga (autotransfusão) (Gusmão et al., 2014). Tanto na medicina como na medicina veterinária, as transfusões sanguíneas ou de hemocomponentes é prática de rotina, entretanto a segurança nessa medida terapêutica nem sempre é garantida, com isso a autotransfusão, um procedimento realizado desde o século passado, vem conquistando espaço, pois reutiliza o sangue do próprio paciente (Gusmão et al., 2014; Kisielewicz e Ian, 2014; Robinson et al., 2016).

O sangue obtido do próprio paciente possui uma série de benefícios, como a ausência das reações imunológicas aos variados tipos sanguíneos e nulidade dos riscos de transmissão de patógenos, (Morikawa et al., 2010; Gusmão et al., 2014; Maglaras et al., 2016). Após coleta e filtragem, é realizada a autotransfusão o mais rapidamente possível (Gusmão et al., 2014; Robinson et al., 2016). Os principais motivos das transfusões sanguíneas são hemorragias, traumas, neoplasias e procedimentos cirúrgicos (Hann et al., 2014; Higgs et al., 2015; Maglaras et al., 2016). Os sinais clínicos apresentados pelo paciente com hemorragia são letargia, palidez das mucosas, aumento no tempo de perfusão capilar (TPC), taquicardia, taquipneia e queda na temperatura corporal, que retornam à normalidade quando recuperada a volemia (Morikawa et al., 2010; Robinson et al., 2016). O objetivo deste relato foi demonstrar a eficiência e a segurança da transfusão sanguínea autóloga em cadela durante laparotomia para hemoperitônio, assim como difundir a técnica e sua aplicação durante situações de emergência.

\section{CASO}

No Hospital Veterinário Universitário, foi atendido um cão da raça Rottweiler, fêmea, com 10 anos de idade, intacta, pesando $42,8 \mathrm{~kg}$, com histórico de nódulo ulcerado na mama abdominal caudal esquerda, medindo 3,3 centímetros de diâmetro, não aderido à musculatura há aproximadamente um ano. Foi realizado o exame físico, constatando-se bom estado geral e variáveis fisiológicas dentro dos limites conhecidos para a espécie: frequência cardíaca (FC), 96 batimentos por minuto (bpm); temperatura corporal $\left(\mathrm{T}^{\circ} \mathrm{C}\right), 38,2^{\circ} \mathrm{C}$; e frequência respiratória $(f), 44$ movimentos por minuto (mpm). Nos exames laboratoriais: hematológico, eritrócitos $6,61 \times 10^{6} / \mu \mathrm{L}$; hemoglobina $14,3 \mathrm{~g} / \mathrm{dL}$; hematócrito 43,0\%; VGM 65,0 fl; CHGM $33,2 \mathrm{~g} / \mathrm{dL}$ e contagem de plaquetas $361 \mathrm{x}$ $10^{3} / \mathrm{mm}^{3}$; bioquímicos: ureia $28,0 \mathrm{mg} / \mathrm{dL}$, creatinina $1,0 \mathrm{mg} / \mathrm{dL}$, alanina aminotransferase (ALT) 57UI/L e proteínas plasmáticas totais $8,0 \mathrm{~g} / \mathrm{dl}$. Diante disso, optou-se pela realização de mastectomia unilateral total e ováriohisterectomia $(\mathrm{OH})$.

Inicialmente, foi fornecida medicação préanestésica (MPA), uma associação de cloridrato de cetamina $(10 \mathrm{mg} / \mathrm{kg})$, midazolam $(0,4 \mathrm{mg} / \mathrm{kg}) \mathrm{e}$ cloridrato de tramadol $(4 \mathrm{mg} / \mathrm{kg})$, todos pela via intramuscular (IM). Depois, a anestesia foi induzida com propofol (dose efeito) e mantida em isoflurano, administrado com vaporizador calibrado e oxigênio a $100 \%$. A fluidoterapia de escolha foi o ringer com lactato $(10 \mathrm{~mL} / \mathrm{kg} / \mathrm{h})$, administrado durante o tempo cirúrgico, quando foram administrados cefalotina $(25 \mathrm{mg} / \mathrm{kg})$, meloxicam $(0,2 \mathrm{mg} / \mathrm{kg})$ e dipirona $(25 \mathrm{mg} / \mathrm{kg})$, todos pela via intravenosa (IV).

Após o procedimento cirúrgico, o animal ficou em observação, permanecendo em decúbito lateral e apresentando FC de 68bpm, $(f)$ de $20 \mathrm{mpm}, \mathrm{T}^{\circ} \mathrm{C}$ de $37,6^{\circ} \mathrm{C}$ e mucosa levemente hipocorada com TPC entre um e dois segundos. Depois de nove horas do término do procedimento cirúrgico, o animal ainda se apresentava em decúbito lateral, com $\mathrm{FC}$ de 110bpm, dispneia, $\mathrm{T}^{\circ} \mathrm{C}$ de $36,2^{\circ} \mathrm{C}$ e mucosa hipocorada com TPC de três segundos, quando se optou pela realização de ultrassonografia abdominal.

Diante da visibilização de líquido livre no abdômen, o animal foi submetido à laparotomia de emergência, e confirmou-se hemorragia abdominal. A coleta do sangue contido na cavidade abdominal foi realizada durante $\mathrm{o}$ procedimento operatório, totalizando aproximadamente $800 \mathrm{~mL}$. O sangue foi transferido de maneira asséptica para uma bolsa de coleta sem a solução anticoagulante de preservação sanguínea (CPDA-1), para evitar possíveis distúrbios de coagulabilidade que esse produto pode acarretar, e foi acoplado a um 
equipo de transfusão com filtro de 180 micrômetros, para filtragem e transfusão imediata, durante o período transoperatório e pós-operatório através da veia cefálica.

Constatou-se hemorragia na ligadura do pedículo ovariano esquerdo, devido à má oclusão dessa ligadura, sendo realizada a hemostasia com fio náilon 2.0. Coágulos foram retirados próximo ao pedículo ovariano esquerdo e descartados; o volume de sangue estimado na cavidade abdominal foi de aproximadamente $1000 \mathrm{~mL}$, sendo $800 \mathrm{~mL}$ transfundidos e $200 \mathrm{~mL}$ descartados por estarem coagulados, aproximadamente $1 / 4$ do volume total de sangue para a espécie.

No pós-operatório, as variáveis clínicas retornaram aos limites fisiológicos, apresentando estado alerta, decúbito esternal após 32 minutos da extubação e mucosa normocorada. A paciente permaneceu internada durante dois dias e recebeu alta para término do tratamento em sua residência. No retorno, realizado seis dias após a autotransfusão, foram detectadas algumas variações esperadas no exame hematológico: eritrócitos $3,52 \times 10^{6} / \mu \mathrm{L}$; hemoglobina $8,5 \mathrm{~g} / \mathrm{dL}$; hematócrito 25,9\%; VGM 73,6fl; CHGM $32,8 \mathrm{~g} / \mathrm{dL}$ e contagem de plaquetas $312 \mathrm{x}$ $103 / \mathrm{mm}^{3}$. Nos exames bioquímicos: ureia $53,6 \mathrm{mg} / \mathrm{dL} ; \quad$ creatinina, $.2 \mathrm{mg} / \mathrm{dL}$; alanina aminotransferase (ALT) 36UI/L e proteínas plasmáticas totais $5,6 \mathrm{~g} / \mathrm{dL}$.

\section{DISCUSSÃO}

Neste relato, foi diagnosticada hemorragia na cavidade abdominal, devido à má oclusão da ligadura do pedículo ovariano, sendo necessária a realização da autotransfusão. A hemorragia na cavidade abdominal pós-cirúrgica é uma das principais causas de transfusão, assim como descrito neste caso (Hann et al., 2014; Higgs et al., 2015; Robinson et al., 2016).

A recuperação do sangue da cavidade abdominal foi realizada pela técnica das duas seringas (Higgs et al., 2015; Robinson et al., 2016). Outros autores descrevem diferentes técnicas de coleta de sangue, com o uso de aparelhos tanto para resgate quanto para transfusão sanguínea (Maglaras et al., 2016; Kellett-Gregory et al., 2017). Já McDevitt et al. (2011) relataram, em seu estudo, que as diferentes técnicas de transfusão não interferem na qualidade do sangue transfundido.

O sangue obtido da paciente foi transferido para uma bolsa de coleta de sangue, onde foi retirada a solução anticoagulante (CPDA-1), de maneira asséptica, já que a solução é desnecessária nesse caso (Rozanski e Laforcade, 2004); o sangue foi filtrado pelo equipo de transfusão, que possui filtro de 180 micrometros, para a retirada de possíveis coágulos, sendo reinfundido através da veia cefálica o mais rapidamente possível (Gusmão et al., 2014; Robinson et al., 2016).

A autotransfusão foi necessária devido à indisponibilidade de sangue no momento do procedimento cirúrgico emergencial, uma vez que esta é praticamente livre de complicações corroborando Robinson et al. (2016), que afirmam que esta é uma técnica segura, utilizada em clínicas e hospitais que não possuem bancos de sangue sem a necessidade de uma grande estrutura.

Outros fatores a serem considerados na escolha de autotransfusão de emergência em relação às transfusões homólogas são: a inexistência de contágio por patógenos e de incompatibilidade de tipo sanguíneo, a redução nos problemas relacionados ao armazenamento e à queda na qualidade do produto, devido ao tempo de armazenamento, e a prevenção da hipotermia, como a reinfusão, pois a temperatura do sangue está próxima da temperatura corporal (Kisielewicz e Ian, 2014; Maglaras et al., 2016). Além dessas, há a vantagem de não haver a necessidade de manter um animal doador, além de equipe técnica treinada disponível para coleta do sangue e preparo do material (Rozanski e Laforcade, 2004).

Os parâmetros fisiológicos observados, as FC e $f$ estavam apresentando um aumento progressivo antes do controle da hemorragia, e, após a reposição volêmica, obtida por meio da autotransfusão, apresentaram decréscimo e estabilização (Morikawa et al., 2010; Hann et al., 2014; Maglaras et al., 2016; Robinson et al., 2016). A temperatura corporal apresentou queda enquanto o paciente estava com hemorragia, retornando aos valores normais após a reposição da volemia (Morikawa et al., 2010; Robinson et al., 2016). 
A despeito das vantagens da autotransfusão, essa técnica tem sido associada a casos de embolismo gasoso, de hemólise, dependendo da pressão aplicada na aspiração do sangue, e ainda tem sido controversa nos casos de neoplasia, pelo risco de transfusão de células neoplásicas (Sikorski et al., 2017; Agboola et al., 2018). A coloração da mucosa antes do início da autotransfusão apresentava-se hipocorada; já ao término, estava normocorada, fato esperado e também relatado por Robinson et al. (2016). O TPC também apresentou melhora significativa, passando de três segundos para um tempo entre um a dois segundos (Morikawa et al., 2010).

Os achados hematológicos observados no retorno da paciente após seis dias foram sugestivos de anemia regenerativa, devido à anisocitose moderada e policromasia discreta, enquanto, na avaliação física, não havia alteração significativa aparente, demonstrando o sucesso do procedimento de emergência adotado, resultados que também foram encontrados por outros autores (Hann et al., 2014; Robinson et al., 2016). A ocorrência de trombocitopenia póscirúrgica, descrita neste caso, pode ser atribuída à grande quantidade de coagulado encontrada na cavidade abdominal ou à trombocitopenia dilucional devido à administração de fluidos durante o procedimento cirúrgico, corroborando Robinson et al. (2016). Este último fato, fluidoterapia, pode justificar a leve redução da proteína plasmática.

Dessa forma, pode-se concluir que a autotransfusão é uma opção econômica e com fácil disponibilidade para casos emergenciais, que pode ser utilizada em locais com poucos recursos, além de evitar a transmissão de patógenos, bem como problemas com incompatibilidade de tipo sanguíneo. Esse procedimento deve ser mais explorado, já que há poucos dados e resultados clínicos descritos na literatura.

\section{REFERÊNCIAS}

AGBOOLA, K.M.; LASALA, J.M.; SINTEK, M.A. et al. Acute right ventricular failure and pulseless electrical activity arrest following autotransfusion of blood. J. Cardiol. Cases, v.17, p.119-122, 2018.
GUSMÃO, L.C.B.; VALÕES, S.H.C.; NETO, J.S.L. Reinfusão transoperatória: um método simples e seguro na cirurgia de emergência. Rev. Coleg. Bras. Cirur., v.41, p.292-296, 2014.

HANN, L.; BROWN, D.C.; KING, L.G. et al. Effect of duration of packed red blood cell storage on morbidity and mortality in dogs after transfusion: 3,095 cases (2001-2010). J. Vet. Intern. Med., v.28, p.1830-1837, 2014.

HIGGS, V.A.; RUDLOFF, E.; KIRBY, R. et al. Autologous blood transfusion in dogs with thoracic or abdominal hemorrhage: 25 cases (2007-2012). J. Vet. Emerg. Crit. Care, v.25, p.731-738, 2015.

KELLETT-GREGORY, L.M.; SETH, M.; ADAMANTOS, S. et al. Autologous canine red blood cell transfusion using cell salvage devices. J. Vet. Emerg. Crit. Care, v.23, p.82-86, 2017.

KISIELEWICZ, C.; IAN, A.S. Canine and feline blood transfusions: controversies and recent advances in administration practices. Vet. Anaesth. Analg., v.41, p.233-242, 2014.

MAGLARAS, C.H.; KOENIG, A.; BEDARD, D.L. et al. Retrospective evaluation of the effect of red blood cell product age on occurrence of acute transfusion-related complications in dogs: 210 cases (2010-2012). J. Vet. Emerg. Crit. Care, v.27, p.108-120, 2016.

MCDEVITT, R.I.; RUAUX, C.G.; BALTZER, W.I. Influence of transfusion technique on survival of autologous red blood cells in the dog. J. Vet. Emerg. Crit. Care, v.21, p.209-216, 2011.

MORIKAWA, M.K.; BOCHIO, M.M.; PINCELLI, V.A. et al. Monitoração e avaliação clínica da eficácia da transfusão de sangue total e concentrado de hemácias em cães. Pesqui. Vet. Bras., v.30, p.665-669, 2010.

ROBINSON, D.A.; KIEFER, K.; QUANDT, J. Autotransfusion in dogs using a 2-syringe technique. J. Vet. Emerg. Crit. Care, v.26, p.766774, 2016.

ROZANSKI, E.; LAFORCADE, A.M. Transfusion medicine in veterinary emergency and critical care medicine. Clin. Tech. Small Anim. Pract., v.19, p.83-87, 2004.

SIKORSKI, R.A.; RIZKALLA, N.A.; YANG, W.W. et al. Autologous blood salvage in the era of patient blood Management. Vox Sanguinis, v.112, p.499-510, 2017. 